\title{
Thermodynamics of spherically symmetric black holes in scale-dependent gravity
}

\author{
P. Bargueño ${ }^{\text {a }}$, J. A. Miralles, J. A. Pons \\ Departamento de Física Aplicada, Universidad de Alicante, Campus de San Vicente del Raspeig, 03690 Alicante, Spain
}

Received: 10 June 2020 / Accepted: 7 December 2020 / Published online: 14 December 2020

(C) The Author(s) 2020

\begin{abstract}
In this work we extend the first law of thermodynamics to spherically symmetric black hole solutions in the context of scale-dependent gravity. After deriving generalized expressions for both the entropy and energy due to the spatial variation of the gravitational constant we analize, by pointing out some relations between scale-dependent and $f(R)$ theories, whether or not the former can be described using equilibrium thermodynamics.
\end{abstract}

\section{Introduction}

Since the pioneering works of Bekenstein [1] and Bardeen et al. [2,3], the connection between gravity and thermodynamics has been continuously growing, being one of the most deep ideas which could put us in the right way towards a microscopical theory of gravity. In particular, by deriving the Einstein equations from both equilibrium thermodynamics and the entropy-area proporcionality for all Rindler horizons, Jacobson [4] pushed forward the idea of treating gravity as en emergent phenomenon whose dynamics could emerge from that of the underlying microscopic degrees of freedom, similarly to the emergence of thermodynamics from statistical mechanics (in particular, this idea has been realized in the context of black holes within loop quantum gravity [5] and the space-time atom [6] perspective [7]). Later, the works of Padmanabhan paved the way for a thermodynamic route to field equations not only in Einstein [8] but also in Lanczos-Lovelock theories [9], showing that the corresponding field equations for a spherically symmetric spacetime can be expressed as $T d S=d E+P d V$. Recently, extensions of Padmanabhan's ideas in $f(R)[10,11], f\left(R, R_{\alpha \beta} R^{\alpha \beta}, \Phi\right)$ [12] and quasi-topological [13] gravities have been performed. Interestingly, Elings, Guedens and Jacobson have shown [14] that, in order to derive the $f(R)$ field equations, a treatment with nonequilibrium thermodynamics is needed,

a e-mail: pedro.bargueno@ua.es (corresponding author) in which an additional entropy production term emerges. Even more, although Cai and Cao have extended this result to scalar-tensor gravity [15], Wu, Yang and Zhang proved [16] that the field equation of Brans-Dicke gravity, scalartensor and $f(R)$ gravity can be derived assuming local thermodynamic equilibrium, provided a careful analysis of the energy which feels the heat flow across the horizon is performed. Later on, the extension of these results to the case of Gauss-Bonnet, $f(\mathcal{G})$ and non-local gravity were performed by Odintsov et al. in [17]. Interestingly, within these extended theories, it is well known that an effective Newton constant appears. Very recently, and in this context, Volovik [18] has assumed a spatially varying Newton constant to be a thermodynamical variable, exploring its consequences for black hole quantization and black hole-white hole quantum tunneling.

Following these ideas, in this work we investigate the role played by a spatially-varying Newton and cosmological constants under the scale-dependent approach [19] in the thermodynamics of spherically symmetric black holes in order to test whether or not the theory can be derived from equilibrium thermodynamics.

This work is organized as follows: Sect. 2 summarizes the main features of scale-dependent gravity which are used in Sect. 3 in order to derive an extended thermodynamical principle in spherically symmetric black holes. The implications of this principle for black hole entropy and energy are discussed in Sect. 4. Finally, after a comparison of the results here reported with that of $f(R)$ theories concerning entropy production are left to Sect. 5, we conclude with some remarks in Sect. 6.

\section{Scale-dependent gravity}

Here we will give a very brief and concise summary of the scale-dependent setting in gravity. For details, the reader is 
referred to Refs. [19,20]. In essence, the idea is to promote the couplings which appears in the gravitational action to scaledependent quantities. Specifically, we start from the scaledependent version of the Einstein-Hilbert action, which is given by

$S\left[g_{\mu \nu}, \tilde{k}\right]=\int \mathrm{d}^{n} x \sqrt{-g}\left[\frac{1}{2 \kappa_{\tilde{k}}}\left(R-2 \Lambda_{\tilde{k}}\right)\right]$,

where $\tilde{k}$ is a scale-dependent field related to a renormalization scale, $\kappa_{\tilde{k}} \equiv 8 \pi G_{\tilde{k}}$ is the Einstein coupling, and $G_{\tilde{k}}$ and $\Lambda_{\tilde{k}}$ refer to the scale-dependent gravitational and cosmological couplings, respectively. As usual, the modified Einstein's equations are obtained by taking variations with respect to the metric field $g_{\mu \nu}$, which lead to

$G_{\mu \nu}+g_{\mu \nu} \Lambda_{\tilde{k}}=-\Delta t_{\mu \nu}$,

where the so-called non-matter energy-momentum tensor, $\Delta t_{\mu \nu}$, is defined as

$\Delta t_{\mu \nu}=G_{\tilde{k}}\left(g_{\mu \nu} \square-\nabla_{\mu} \nabla_{\nu}\right) G_{\tilde{k}}^{-1}$.

In addition, by taking the variation of the effective action with respect to the scale field, $\tilde{k}(x)$, one imposes

$\frac{\mathrm{d}}{\mathrm{d} \tilde{k}} S\left[g_{\mu \nu}, \tilde{k}\right]=0$,

which can be seen as an a posteriori condition towards background independence.

In essence, both Eqs. (2) and (4) close the system. The problem is that the $\beta$-functions, which describe the renormalization group running of both $G_{\tilde{k}}$ and $\Lambda_{\tilde{k}}$ are, in general, unknown (in fact they depend of how a specific problem is solved). Therefore, in order to avoid this issue, we consider that both couplings, $G_{\tilde{k}}$ and $\Lambda_{\tilde{k}}$, inherit the dependence on the space-time coordinates from the space-time dependence of the scale field, $\tilde{k}(x)$. In this sense, the couplings are written as $G(x)$ and $\Lambda(x)$. This idea, together with an appropriate choice for the line element, allows, in principle, to solve the problem in situations with a high degree of symmetry.

With these ideas in mind, scale-dependent gravity can be considered, in some sense, as a special kind of scalar-tensor theory, although the improved action is not supposed to be varied neither with respect to $G(x)$ nor to $\Lambda(x)$ [19]. The total action we consider is

$S_{\mathrm{T}}=\int d^{4} x \sqrt{-g}\left(\frac{R}{16 \pi G(x)}-\frac{\Lambda(x)}{8 \pi G(x)}\right)+S_{\mathrm{mat}}$,

where $S_{\text {mat }}$ encodes the matter sector of the theory.

Then, after variating Eq. (5) with respect to the metric, we arrive to

$G_{\alpha \beta}+G\left(g_{\alpha \beta} \square-\nabla_{\alpha} \nabla_{\beta}\right) G^{-1}+\Lambda g_{\alpha \beta}=8 \pi G T_{\alpha \beta}$ or

$\mathcal{S}_{\alpha \beta} \equiv G_{\alpha \beta}+\Delta t_{\alpha \beta}+\Lambda g_{\alpha \beta}-8 \pi G T_{\alpha \beta}=0$,

where, as stated before, $G$ and $\Lambda$ have to be understood as $G(x)$ and $\Lambda(x)$.

At this point, a couple of important points are in order. First, let us note that, after variations of the scale field, Eq. (4) turns out to be

$$
\left(R \nabla_{\mu}\left(\frac{1}{G_{\tilde{k}}}\right)-2 \nabla_{\mu}\left(\frac{\Lambda_{\tilde{k}}}{G_{\tilde{k}}}\right)\right) \partial^{\mu} \tilde{k}=0 .
$$

And second, this equation, together with Eq. (2), are consistent with diffeomorphism invariance, as expressed by the conserved covariance of the Einstein tensor, as explicitly shown in [20].

In addition, let us note that the Einstein tensor is covarianty conserved and, therefore, $\nabla^{\mu}\left(g_{\mu \nu} \Lambda(r)+\Delta t_{\mu \nu}(r)-\right.$ $\left.8 \pi G(r) T_{\mu \nu}\right)$ vanishes on shell, as can be explicitly shown. Therefore, consistency is guaranteed also at this level.

\section{Generalized Einstein equations as a thermodynamic identity}

In principle, in view of Eq. (7), one is tempted to assign some kind of energy densities and pressures associated with the non-matter energy-momentum tensor, by interpreting it as some kind of curvature fluid, as is usually established in $f(R)$ theories [21]. In this section, following closely Padmanabhans's ideas [8], we will show that the interpretation of the $\Delta t_{\alpha \beta}$ terms is very different to that of the usual matter sector, $T_{\alpha \beta}$, and that it gives place to an extended thermodynamics of spherically symmetric black holes.

Let us consider a line element written in Schwarzschild coordinates as

$d s^{2}=-f(r) d t^{2}+f(r)^{-1} d r^{2}+r^{2} d \Omega^{2}$,

where $d \Omega^{2}$ is the line element four the round two-sphere. ${ }^{1}$

Even more, within this symmetry, $\Lambda$ and $G$ turn into functions of only the radial coordinate. Therefore, the generalized radial equation can be written as

$\frac{1}{r^{2}}\left(1-f-r^{2} \Lambda\right)-\frac{f^{\prime}}{r}=-G T_{r}^{r}-\frac{G^{\prime}}{G}\left(\frac{f^{\prime}}{2}+\frac{2 f}{r}\right)$,

where the prime denotes derivative with respect to the radial coordinate, $r$.

\footnotetext{
${ }^{1}$ As shown in [8], one can extend much of this analysis to a more general case where $g_{t t}=-f(r)$ and $g_{r r}=g(r)$ with $f \neq g$ in a fairly straightforward manner.
} 
Let us particularize to the case of an event horizon located at $r_{+}=a$. Then, $f(a)=0$. In this case we get

$$
f^{\prime}(a)=\left(\frac{1}{a}-a\left(\Lambda(a)-T_{r}^{r}(a) G(a)\right)\right)\left(1-\frac{a}{2} \frac{G^{\prime}(a)}{G(a)}\right)^{-1} \equiv B
$$

or

$\frac{1}{2} B a-\frac{1}{2}=\frac{1}{2} T_{r}^{r}(a) G(a) a^{2}-\frac{\Lambda(a)}{2} a^{2}+\frac{1}{4} B \frac{G^{\prime}(a)}{G(a)} a^{2}$.

Multiplying by $d a$ and dividing by $G(a)$ in both sides, we arrive to

$\frac{B}{4 \pi} d\left(\frac{\pi a^{2}}{G(a)}\right)-\frac{d a}{2 G(a)}=\left(T^{r}{ }_{r}(a)-\frac{\Lambda(a)}{8 \pi G(a)}\right) 4 \pi a^{2} d a$

This expression, Eq. (13), is our main result. Interestingly, it can be written as

$d E=T d S-[P+P(\Lambda, G)] d V$,

where

$d E=\frac{d a}{2 G(a)}$

is the energy change within scale-dependent gravity during a infinitesimal horizon displacement, $d a$,

$V=\frac{4 \pi a^{3}}{3}$

is the areal volume,

$T=\frac{f^{\prime}(a)}{4 \pi}$

is the usual Hawking temperature (which can be obtained from the periodicity consideration of the Euclidean time by Wick rotating the time coordinate $t \rightarrow i \tau$. The metric will be regular at the horizon if $\tau$ is taken to be an angular variable with a period $\beta=4 \pi / f^{\prime}(a)$, which is just the inverse Hawking temperature $T=1 / \beta$ of the black hole. Therefore, the Hawking temperature only depends of the specific gravitational theory we are dealing with through the specific form of $f(r))$,

$S=\frac{A}{4 G(a)}$

is the generalized Bekenstein-Hawking entropy (where $A$ is the area of the event horizon),

$P=T_{r}^{r}(a)$

is a pressure term due to the matter sector (evaluated at the horizon) and

$P(\Lambda, G)=-\frac{\Lambda(a)}{8 \pi G(a)}$

is the pressure due to the position-dependent constants evaluated at the horizon, $\Lambda(a)$ and $G(a)$. Note that the usual general relativistic definitions and relations of Ref. [8] are obtained in the limit $\{G(r), \Lambda(r)\} \rightarrow\{\Lambda, G\}$.

Here we note that the expression for the scale-dependent black hole entropy, which we remind the reader is given by

$S=\frac{A}{4 G(a)}$,

formally coincides with that of Brans-Dicke theory [22]. In this sense, there is no ambiguity when computing black hole entropies in the approach here employed, as we will point out in the following section.

\section{Implications for black hole entropy and energy}

As have been noticed several times during the last years (see, for example, [23]), the improved gravitational equations in a spherically symmetric $(1+3)$-dimensional spacetime have to be suplemented with an additional constraint in order for the system to be closed. This is due mainly to the necessity of including the cosmological constant in order not to obtain trivial (i.e., pure general relativistic) solutions. In this case, we have four unknowns: two metric potentials plus $G(r)$ and $\Lambda(r)$ but only three equations: $\mathcal{S}_{t t}, \mathcal{S}_{r r}$ and $\mathcal{S}_{\theta \theta}$ (or $\mathcal{S}_{\phi \phi}$ ). The required aditional information is usually introduced by means of an appropriate ansantz which reduces from two to one metric potentials by the usual relation $g_{t t} g_{r r}=-1$ [23].

Specifically, let us consider a particular solution to Eq. (7) with an ansantz given by Eq. (9). As pointed out by Jacobson [24], for any spherically symmetric and static geometry with $g_{t t}=-f(r)$ and $g_{r r}=g(r)$ we have that

$R_{\alpha \beta} n^{\alpha} n^{\beta}=\frac{(f g)^{\prime}}{r g}$

where $n$ is any radial null vector.

Therefore, if the matter sector satisfies

$T_{\alpha \beta} n^{\alpha} n^{\beta}=0$

then, Eq. (7) together with Eq. (9) imply

$\Delta t_{\mu \nu} n^{\alpha} n^{\beta}=0$

which, for the case we are interested in, reduces to

$2\left(G^{\prime}\right)^{2}=G G^{\prime \prime}$

which is solved by

$G(r)=\frac{G_{0}}{1+\frac{r}{l_{\mathrm{S}}}}$,

where the two constants of integration have been chosen in order to recover the usual general relativistic expression when 
the running scale, $l_{\mathrm{S}}$, which signals the region where scaledependent effects are supposed to appear, goes to infinity and $G(r) \rightarrow G_{0}{ }^{2}$

Interestingly, this explicit form of the Newton coupling is valid not only for the vacuum case (improved Schwarzschild(anti)-de Sitter solution [25]) but also for several electrovacuum solutions, including the improved Reissner-Nördstrom one [26], politropic [27], regular [28], planar [29] and rotating black holes [30], where Eq. (23) is satisfied.

Regarding the behaviour of $\Lambda(r)$, it is interesting to note that the divergence of Eq. (6) implies

$\Lambda^{\prime}+\frac{1}{8 \pi G} \frac{G^{\prime}}{G}\left(\frac{R}{2}-\Lambda\right)=p^{\prime}+(\rho+p) \frac{f^{\prime}}{2 f}$,

which is the generalized Tolman-Oppenheimer-Volkoff equation when both $G$ and $\Lambda$ are functions of the radial coordinate. As usual, the mixed components of the energymomentum tensor of the matter sector are (assuming isotropy and spherical symmetry) $\operatorname{diag}(-\rho, p, p, p)$.

If the vacuum case is considered we note that, taking into account Eqs. (26), (27) can be formally solved as

$\Lambda(r)=e^{-r /\left(8 \pi G_{0} l_{\mathrm{S}}\right)}\left(\Lambda_{0}-\frac{1}{16 \pi G_{0} l_{\mathrm{S}}} \int_{1}^{r} e^{-y /\left(8 \pi G_{0} l_{\mathrm{S}}\right)} R(y) d y\right)$,

which goes to $\Lambda_{0}$ in the $l_{\mathrm{S}} \rightarrow \infty$ limit. Interestingly, if the integral term could be taken to be negligible for a particular solution, Eq. (28) shows an exponential decay of the effective cosmological constant.

With the explicit expression for $G(r)$ at hand, now it is straightforward to calculate the entropy in the scaledependent framework, derived in Sect. 3. We get

$S=\frac{A}{4 G(a)}=\frac{\pi a^{2}}{4 G_{0}}\left(1+\frac{a}{l_{\mathrm{S}}}\right)$,

which is the expression usually employed in scale-dependent improved black hole solutions [25-30].

It is worth noting that Eq. (29) can be written as

$S=\frac{A}{4 G(a)}=\frac{1}{4 G_{0}}\left(A+\frac{3}{4} \frac{V}{l_{\mathrm{S}}}\right)=S_{0}+\frac{3}{16 G_{0}} \frac{V}{l_{\mathrm{S}}}$,

showing the appereance of a "volumetric component" for the entropy modulated by the running scale, in addition to the usual area term, $S_{0}$.

Concerning the energy, defined in our approach as

$E=\int \frac{d a}{2 G(a)}$,

we get, for the clase of black holes here considered,

$E=E_{0}+\frac{a^{2}}{4 G_{0} l_{\mathrm{S}}}$,

$\overline{2}$ Equation (25) can also de obtained from $\Delta t^{t}{ }_{t}=\Delta t^{r}{ }_{r}$ in order to have consistency with the Schwarzschild ansantz given by Eq. (9). where $E_{0}=a / 2 G_{0}$ is the non-running energy which, for example, coincides with the Misner-Sharp mass for a Schwarzschild black hole. Even more, by the Smarr law we have $E_{0}=2 T S_{0}$ and, therefore, the corrected energy satisfies an extended Smarr-like law as

$E=2\left(T+T_{l_{\mathrm{S}}}\right) S_{0}$,

where we have defined $T_{l_{\mathrm{S}}}=\frac{1}{8 \pi l_{\mathrm{S}}}$ (note its similarity with the Hagedorn temperature of strings).

\section{Comparison with other approaches}

As commented in the Introduction, there have been some discussion on the possibility that both $f(R)$ and scalar-tensor theories were described by non-equilibrium or equilibrium thermodynamics $[10,14,15]$. Specifically, in spherical symmetry, the radial gravitational equation in $f(R)$ gravity can be written, using our notation, as [10]

$$
\begin{aligned}
& \frac{B}{4 \pi} d\left(\frac{\pi a^{2} F(R)}{G_{0}}\right)-F(R) \frac{d a}{2 G_{0}} \\
& =T^{r}{ }_{r}(a) 4 \pi a^{2} d a+\frac{1}{4 G_{0}}(f(R)-R F(R)) a^{2} d a,
\end{aligned}
$$

where $F(R) \equiv \frac{d f(R)}{d R}$ and $R$ has to be understood as evaluated at the horizon, $R(a)$. Then, Eq. (34) can be written as

$d E=T(d S+d \bar{S})-P d V$,

where, in analogy with our case, $d E=\frac{F(R) d a}{2 G_{0}}$ and $S=$ $\frac{F(R) A}{4 G_{0}}$ are the energy change and entropy, respectively, $P=$ $T^{r}{ }_{r}(a)$ is the corresponding pressure due to matter terms (note that, in Ref. [10], the cosmological constant is not taken into account) and $\frac{B}{4 \pi}$ is the Hawking Temperature. The new term, which is not present in the scale-dependent setting, is the entropy production, given by

$d \bar{S}=T \frac{A}{4 G_{0}} \frac{f(R)-R F(R)}{B} d a$.

Note that the third term of the rhs of Eq. (12) goes with the temperature, which permits to include it into the $T d S$ term. On the contrary, the second term of the rhs of Eq. (34) does not have it and, therefore, an entropy production has to be included. Interestingly, the authors of Ref. [10] argued that it is reasonable to redefine the energy associated with the black hole horizon as $d \bar{E}=d E+\frac{f(R)-R F(R)}{4 G_{0}} a^{2} d a$ instead of introducing an entropy-production term, which is in agreement with the ideas presented in [16] regarding a redefinition of the energy which feels the heat flux in order to mantain equilibrium thermodynamics. 
If this last point of view is adopted, we note that we have complete analog definitions with that of $f(R)$, i.e.:

$$
\begin{aligned}
& E=\int \frac{F(R)}{2 G_{0}} d a \leftrightarrow \int \frac{d a}{2 G(r)} \\
& S=\frac{F(R) A}{4 G_{0}} \leftrightarrow \frac{A}{4 G(a)} .
\end{aligned}
$$

Therefore, scale-dependent and $f(R)$ gravities are equivalent at this level when $F(R)$ is identified with $\frac{G_{0}}{G(r)} \cdot{ }^{3}$ At this point, a number of points are in order. First; note that the value of $G(r)$ given by Eq. (26) is a consequence of the ansatz given by Eq. (9). In fact, $G(r)$ is independent of the metric for this specific ansantz, as Eq. (25) shows. Therefore, $G(r)$ can not be considered a function of $R$, since $R$ explicitly depends on the metric choice. Second; in this sense, $F(R)=$ $\frac{G_{0}}{G(r)}=$ constant, and, therefore, $f(R)-R F(R)=0$ without having $f(R)=R$. Third; under this equivalence, now it is easy to show that scale-dependent gravity can be understood as a consequence of equilibrium thermodynamics, withouth having to add neither entropy production nor modificacions to the energy, due to the vanishing of the term $f(R)-R F(R)$ (the underlying theory is not general relativity but a special case of $f(R, \Phi)$ theories with $f(R, \Phi)=\Phi R=G(x)^{-1} R$, where $\Phi$ is taken to be non-dynamical, i.e., there are not kinetic terms in the action). These facts make our findings to be in complete agreement with general claims concerning scalar-tensor and $f(R)$ theories [16].

\section{Conclusions}

In this work we have derived a thermodynamic formulation for scale-dependent gravity in spherically symmetric black holes. After obtaining appropriate generalizations for both the entropy and energy of scale-dependent black holes, we have shown that no entropy production terms are needed in order to describe the theory, in complete agreement with both scalar-tensor and $f(R)$ theories.

Acknowledgements We acknowledge support from the Spanish Agencia Estatal de Investigación (grant PGC2018-095984-B-I00) and Generalitat Valenciana (grant PROMETEO/2019/071). P. B. is funded by the Beatriz Galindo contract BEAGAL 18/00207 (Spain). P. B. dedicates this work to Anaís, Lucía, Inés and Ana for continuous support.

Data Availability Statement This manuscript has no associated data or the data will not be deposited. [Authors' comment: The present work is a theoretical study, and therefore no experimental data has been employed.]

Open Access This article is licensed under a Creative Commons Attribution 4.0 International License, which permits use, sharing, adaptation, distribution and reproduction in any medium or format, as long as you

\footnotetext{
${ }^{3}$ Here we refer to scale-dependent gravity without cosmological constant.
}

give appropriate credit to the original author(s) and the source, provide a link to the Creative Commons licence, and indicate if changes were made. The images or other third party material in this article are included in the article's Creative Commons licence, unless indicated otherwise in a credit line to the material. If material is not included in the article's Creative Commons licence and your intended use is not permitted by statutory regulation or exceeds the permitted use, you will need to obtain permission directly from the copyright holder. To view a copy of this licence, visit http://creativecomm ons.org/licenses/by/4.0/.

Funded by $\mathrm{SCOAP}^{3}$.

\section{References}

1. J.D. Bekenstein, Phys. Rev. D 7, 2333 (1973)

2. J.M. Bardeen. B. Carter, S.W. Hawking. Commun. Math. Phys. 31, 161 (1973)

3. S.W. Hawking, Commun. Math. Phys. 43, 199 (1975)

4. T. Jacobson, Phys. Rev. Lett. 75, 1260 (1995)

5. A. Ghosh, K. Noui, A. Perez, Phys. Rev. D 89, 084069 (2014)

6. T. Padmanabhan, Int. J. Mod. Phys. D 19, 2275 (2010)

7. A.F. Vargas, E. Contreras, P. Bargueno, Gen. Relativ. Gravity 50, 117 (2018)

8. T. Padmanbhan, Class. Quantum Gravity 19, 5387 (2002)

9. A. Paranjape, S. Sarkar, T. Padmanabhan, Phys. Rev. D 74, 104015 (2006)

10. M. Akbar, R.G. Cai, Phys. Lett. B 648, 243 (2007)

11. Y. Zheng, R. Yang, Eur. Phys. J. C 78, 682 (2018)

12. M. Zubair, F. Kousar, S. Bahamonde, Phys. Dark. Univ. 14, 116 (2016)

13. A. Sheyki, M.H. Dehghani, R. Dehghani, Gen. Relativ. Gravity 46, $1679(2014)$

14. C. Elings, R. Guedens, T. Jacobson, Phys. Rev. Lett. 96, 121301 (2006)

15. R.G. Cai, L.M. Cao, Phys. Rev. D 75, 064008 (2007)

16. S.F. Wu, G.H. Yang, P.M. Zhang, Prog. Theor. Phys. 120, 615 (2008)

17. K. Bamba, C.-Q. Geng, S. Nojiri, S.D. Odintsov, EPL 89, 50003 (2010)

18. G. E. Volovik, (2020). arXiv:2003.10331v6

19. M. Reuter, H. Weyer, Phys. Rev. D 69, 104022 (2004)

20. B. Koch, P. Rioseco, C. Contreras, Phys. Rev. D 91, 025009 (2015)

21. S. Capozziello, V. Faraoni, Beyond Einstein Gravity: A Survey of Gravitational Theories for Cosmology and Astrophysics (Springer, New York, 2011)

22. B. Koch, I.A. Reyes, Á. Rincón, Class. Quantum Gravity 33, 225010 (2016)

23. Á. Rincón, B. Koch, J. Phys. Conf. Ser. 1043, 012015 (2018)

24. T. Jacobson, Class. Quantum Gravity 24, 5717 (2007)

25. C. Contreras, B. Koch, P. Rioseco, Class. Quantum Gravity 30, 175009 (2013)

26. B. Koch, P. Rioseco, Class. Quantum Gravity 33, 035002 (2016)

27. E. Contreras, Á. Rincón, B. Koch, P. Bargueno, Eur. Phys. J. C 78, $246(2018)$

28. E. Contreras, Á. Rincón, B. Koch, P. Bargueno, Int. J. Mod. Phys. D 27, 1850032 (2018)

29. Á. Rincón, E. Contreras, P. Bargueno, Eur. Phys. J. Plus 134, 557 (2019)

30. E. Contreras, Á. Rincón, G. Panotopoulos, P. Bargueno, B. Koch, Phys. Rev. D 101, 064053 (2020) 